\title{
Extended resection for seizure control of pure motor strip focal cortical dysplasia during awake craniotomy: illustrative case
}

\author{
Bayron A. Sandoval-Bonilla, MD, MSc, ${ }^{1}$ André Palmini, MD, PhD, ${ }^{2}$ Eliseu Paglioli, MD, ${ }^{2}$ Alejandro Monroy-Sosa, MD, ${ }^{3,4}$ \\ Maria F. De la Cerda-Vargas, MD, ${ }^{5}$ Job J. Rodríguez-Hernández, MD, ${ }^{1}$ Victor R. Chávez-Herrera, MD, ${ }^{1}$ Sara P. Perez-Reyes, MD, MSc, ${ }^{6}$ \\ Fernando C. Castro-Prado, MD, PhD, ${ }^{1,7}$ Samuel Perez-Cardenas, MD, ${ }^{8}$ Josafat J. Sánchez-Dueñas, PhD, ${ }^{1}$ and \\ Lucero N. Lagunes-Padilla, $\mathrm{MD}^{9}$
}

\begin{abstract}
${ }^{1}$ Department of Neurosurgery, Epilepsy Surgery Program, Hospital de Especialidades, CMN Siglo XXI, Instituto Mexicano del Seguro Social, Mexico City, Mexico; ${ }^{2}$ Department of Neurology and Neurosurgery, Epilepsy Surgery Program, Hospital São Lucas da Pontificia Universidade Católica do Rio Grande do Sul (PUCRS), Porto Alegre, Brazil; ${ }^{3}$ Department of Neurosurgery, Aurora Neuroscience Innovation Institute, Aurora St. Luke's Medical Center, Milwaukee, Wisconsin; ${ }^{4}$ Skull Base, Brain \& Cerebrovascular Laboratory, Advocate Aurora Research Health Institute, Milwaukee, Wisconsin; ${ }^{5}$ Department of Neurosurgery, Hospital de Especialidades No. 71, Instituto Mexicano del Seguro Social, Torreón Coahuila, Mexico; ${ }^{6}$ Department of Neurosurgery, Hospital Regional de Alta Especialidad del Bajío, Instituto Mexicano del Seguro Social, Leon, Guanajuato, Mexico; ${ }^{7}$ Department of Neurosurgery, High Specialties Regional Hospital Gral. I. Zaragoza, ISSSTE, Mexico City, Mexico; ${ }^{8}$ Department of Neurosurgery, University Hospital Monterrey, Nuevo Leon, Mexico; and ${ }^{9}$ Department of Neurosurgery, Hospital Angeles Universidad, Mexico City, Mexico
\end{abstract}

BACKGROUND Focal cortical dysplasias (FCD) represent highly intrinsically epileptogenic lesions that require complete resection for seizure control. Resection of pure motor strip FCD can be challenging. Effective control of postoperative seizures is crucial and extending the boundaries of resection in an eloquent zone remains controversial.

OBSERVATIONS The authors report a 52-year-old right-handed male with refractory epilepsy. The seizure phenotype was a focal crisis with preserved awareness and a clonic motor onset of right-hemibody. Epilepsy surgery protocol demonstrated a left pure motor strip FCD and a fullawake resective procedure with motor brain mapping was performed. Further resection of surgical boundaries monitoring function along intraoperative motor tasks with no direct electrical stimulation corroborated by intraoperative-neuromonitorization was completed as the final part of the surgery. In the follow-up period of 3-years, the patient has an Engel-IB seizure-control with mild distal lower limb palsy and no gate compromise.

LESSONS This report represents one of the few cases with pure motor strip FCD resection. In a scenario similar to this case, the authors consider that this variation can be useful to improve seizure control and the quality of life of these patients by extending the resection of a more extensive epileptogenic zone minimizing functional damage.

https://thejns.org/doi/abs/10.3171/CASE21605

KEYWORDS focal cortical dysplasia; epilepsy surgery; brain mapping; awake craniotomy

Resection of intrinsic brain lesions within or closely adjacent to eloquent areas demands functional mapping to avoid impairment or damage. ${ }^{1,2}$ Intraoperative brain mapping is the standard technique to identify cortical function and white matter connectivity, based on the assumption that function can be elicited or temporarily interrupted by direct electrical stimulation. ${ }^{1,2}$ Not infrequently, resection is halted in the proximity of a functional zone because subcortical mapping may be unreliable close to any fiber tract. ${ }^{2-4}$ In this situation, the surgeon has to decide if further resection is undertaken in the proximity or through a tract and thus determines to conclude the procedure. ${ }^{5}$ Therefore, a delicate balance between the extent of resection and risk of functional deficit with impact upon quality of

ABBREVIATIONS BOLD = blood oxygenation level dependent; DTI = diffusion tensor imaging; ECoG = electrocorticography; EEG = electroencephalogram; FCD = focal cortical dysplasia; $\mathrm{fMRI}$ = functional magnetic resonance imaging; IONM = intraoperative-neuromonitorization; $M B M=$ motor brain mapping; $M E P=$ motor evoked potential; $M R I=$ magnetic resonance imaging; MTD = motor task device; PMA = primary motor area. INCLUDE WHEN CITING Published March 7, 2022; DOI: 10.3171/CASE21605.

SUBMITTED October 21, 2021. ACCEPTED January 3, 2022.

(C) 2022 The authors, CC BY-NC-ND 4.0 (http://creativecommons.org/licenses/by-nc-nd/4.0/). 
life must be stricken in such scenarios, and the final decision must be individualized.

Because favorable surgical results in focal cortical dysplasia (FCD) depend on complete resection of the lesion, involvement of the motor strip creates a particular challenge. ${ }^{6}$ Our objective is to describe a case of a pure motor strip FCD during awake craniotomy with an alternative approach that may circumvent some of the uncertainties related to functional mapping in this region.

\section{Illustrative Case}

A 52-year-old right-handed male with refractory seizures and no known risks factors was referred for evaluation. Seizures began when he was 18 months old and continued with a high frequency throughout his life. He could have 15 to 20 daily seizures manifested by focal motor clonic movements involving the right hemibody with progressive impairment of cognition. Neurological examination was normal. Interictal electroencephalogram (EEG) had bilateral frontotemporal discharges and in the context of a comprehensive presurgical epilepsy surgery evaluation protocol, a video-EEG showed left frontal ictal discharges. Magnetic resonance imaging (MRI) showed a typical FCD with a transmantle sign in the left motor cortex. Functional magnetic resonance imaging (fMRI) and diffusion tensor imaging (DTI) tractography delineated the primary motor area (PMA) and pyramidal tract trajectory, respectively. An awake craniotomy with intraoperative motor brain mapping (MBM) was proposed by the Epilepsy Surgery Multidisciplinary Group as the most suitable resection strategy in this patient with an epileptogenic dysplastic lesion within the primary motor cortex, yet without motor deficits.

A standard navigation-guided craniotomy centered to the central lobe was performed under a full-awake anesthesia technique. Dura mater was widely opened in a C-shaped fashion to allow access to the dorsolateral surface of the central lobe. Anatomical and functional structures were identified and marked on cortical surface. The brain mapping protocol performed in our center includes fusion of blood oxygenation level-dependent (BOLD) images of the PMA obtained in the fMRI with the pyramidal tract DTI and the structural lesion in the navigation system (BrainLab) as a guidance before direct stimulation. Cortical stimulation is regularly performed under conventional Penfield parameters $(60 \mathrm{~Hz}$ bipolar stimuli with a pulse duration of $0.3 \mathrm{msec}$ starting at $1 \mathrm{~mA}$ with a subsequent increment of $1 \mathrm{~mA}$ until reaching a positive motor reaction) to corroborate PMA localization ${ }^{2}$ previously delineated by BOLD images added in the navigation system. After an initial central FCD resection with subpial technique, dynamic subcortical stimulation and resection were conducted with a modification of Taniguchi parameters according to the size of the lesion (monopolar train of 5 pulses stimuli starting on a current of $10 \mathrm{~mA}$ with a gradual modification of $1 \mathrm{~mA}$ ) seeking to identify the pyramidal tract ${ }^{2}$ with a suction-probe device (Inomed) also adapted to the navigation system. The final part of the resection, the circumferential margin of the cavity, was conducted with close monitoring of motor evoked potentials (MEP; Caldwell) by $\mathrm{IONM}^{7}$ at regular intervals of 1 to 3 minutes plus a visual qualitative performance of tapping, a continuum medial rotation-cubital deviation movement of the upper limb with an ad hoc device designed and developed at our center and distal flexion-extension movements of the right extremities. This final segment of the resection was not guided by MBM but by an in situ qualitative functional feedback regularly confirmed with quantitative MEP monitoring (a $<50 \%$ reduction of amplitude regarding a basal recording previous to FCD resection is the optimal goal to conclude the resection to avoid a permanent deficit). Electrocorticography (ECoG) was used to confirm complete resection of epileptogenic abnormalities during the procedure as a relevant tool of the decision-making process to extend resection margins. $^{8,9}$ Histopathology confirmed a type II FCD, and the patient obtained an Engel IB outcome (2 to 4 seizures per year during the last 42 months). Transient moderate lower limb and mild upper limb paresis eventually recovered almost completely 4 months after operation as expected (Fig. 1).

\section{Discussion}

\section{Observations}

Conventional MBM accurately demonstrated that the FCD was located in the junction of the right upper and lower limb representation of the left motor strip. Structural and functional neuronavigation efficiently localized lesional margins and functional zones. fMRI can also be associated to the navigation system together with tractrography as an effective intraoperative guidance before MBM.

ECoG demonstrated that further resection beyond structural margins was required to obtain a complete resection of the epileptogenic zone.

An additional resection of surgical boundaries with no direct electrical stimulation while the function is monitored with intraoperative motor tasks and corroborated with IONM at regular intervals to complete the resection, provides the surgeon a safe ECoG-guided extension of surgical margins for seizure control.

\section{Lessons}

The goal of the surgery was to perform a gross total FCD resection plus an ECoG-guided extended resection with the awareness that the patient may have a motor postoperative deficit to obtain effective seizure control. Intraoperative motor function changes were expected based on the approach performed at the end of the resection using an ad hoc motor task device (MTD).

The ultimate purpose of brain mapping is to identify eloquent zones avoiding functional impairment during a resective procedure. $^{10,11}$ Direct electrical stimulation is the gold standard technique. ${ }^{2,12}$ Extensive series have been published including lesions that include or are adjacent to the motor strip. ${ }^{10,13-15}$ There are few reports concerning pure motor strip surgery because it implies some degree of functional deficit and a potentially unacceptable outcome. $^{10,11,16,17}$

FCD are highly intrinsically epileptogenic lesions that require complete resection for seizure control. ${ }^{18-21}$ Refractory epilepsy can generate a significant cognitive impairment and a negative impact on the quality of life of these patients. Therefore, we consider surgical resection of FCDs even if the lesion is located in the motor eloquent cortex. A delicate balance between a pure motor strip FCD resection and preservation of function to minimize the damage should be achieved because an effective control of postoperative seizures is crucial. ${ }^{14,22,23}$

Given that surrounding cortex may contribute to the epileptogenic zone of an MRI-visible FCD, a more extensive epileptogenic zone requires more extensive resection. ${ }^{8,24}$ Favorable seizure control can also be obtained with pure lesionectomy and for this reason every case must be intraoperatively evaluated with ECOG to decide extending the resection if possible.,

When resecting lesions adjacent to the motor strip, cortical and subcortical mapping, respectively, identify PMA and the pyramidal 

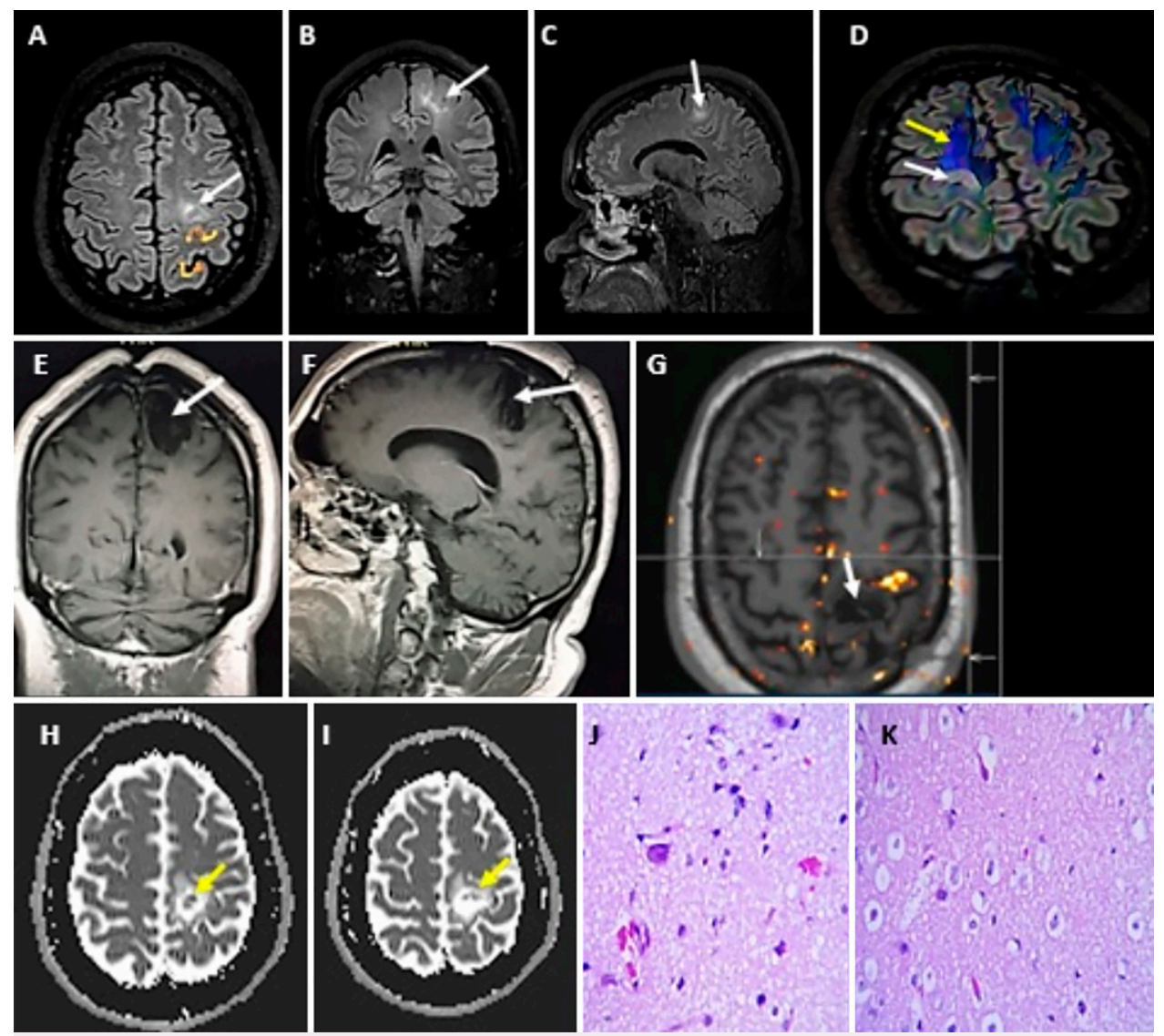

FIG. 1. Presurgical neuroimaging findings. A: fMRI, hyperdensity signal in left precentral gyrus corresponding with a FCD (white arrow) adjacent to left hand motor area. B and C: Coronal and sagittal flair-weighted MRI showing a cortical hyperintense image in the bottom of precentral sulcus corresponding to the FCD (white arrow). D: Pyramidal tract (yellow arrow) close to FCD (white arrow). Postsurgical neuroimaging findings. E and F: Sagittal and coronal T1-weighted MRI showing the area of FCD resection (white arrow). G: fMRI 1 year after surgery. FCD resection area (white arrow) adjacent to the left-hand motor region is observed. $\mathrm{H}$ and I: Axial diffusion-weighted MRI, 1 year after surgery. No evidence of infarcts adjacent to the FCD resection area (yellow arrows). J and K: Histopathological findings, hematoxylin-eosin staining, showing type II FCD (microscopic heterotopia outside of cortical layer I).

tract. $^{15,25-27}$ This enables the surgeon to access through a safe entry site restricted only to the FCD avoiding additional functional compromise. Safe subcortical identification of fibers requires approximately 5 to $10 \mathrm{~mm}$ of distance from the stimulation probe to ensure functional preservation (1.0 to $1.5 \mathrm{~mA} / 1 \mathrm{~mm})^{2,4,5,28}$ This is particularly evident with bottom of sulcus dysplasias (BOLD) or superficial FCD in the proximity of eloquent zones. Irrespective of bipolar or monopolar stimulation, there is a certain proximity $(1$ to $3 \mathrm{~mm}$ ) in which direct electrical stimulation does not guarantee functional safety and this is particularly evident within a functional area. ${ }^{1}$ Within the PMA, both conventional MBM and neuronavigation efficiently guide a lesionectomy. Nevertheless, in such cases extending resection borders with a minimal functional deficit faces a challenge given that there is no safe distance to be identified and preserved.

Direct electrical stimulation for MBM can be performed either with a probe or a strip placed over the PMA surface. In both cases, electrical current is applied during a specific period (2- to 4-second average) at regular intervals. Within PMA, direct electrical stimulation elicits a response that represents a potential bias caused by involuntary movements and artifacts on electromyography monitoring during resection. To solve this, in case in which a more extensive epileptogenic zone is identified by $E C O G,{ }^{8,9}$ we consider further resection of surgical boundaries guided by the monitoring of function with intraoperative motor tasks confirmed with IONM at regular intervals ${ }^{7}$ with no direct electrical stimulation at the end of the resection.

This technique is different from previously described approaches for awake resections of lesions in the motor cortex because we use an ad hoc MTD. MTD was designed to optimize the testing time related to the dorsolateral margin of the resection which corresponds to the cortical upper limb representation and subcortical fibers of the pyramidal tract. This instrument was planned to perform a continuum medial rotation-cubital deviation movement of the upper limb that combines the qualitative monitoring of distal and proximal muscles in a short period and allows early detection of subtle motion compromise. The MTD was manufactured to be compatible with fMRI scans (Fig. 2). Resection corresponding to the surgical margin of cortical lower limb representation 

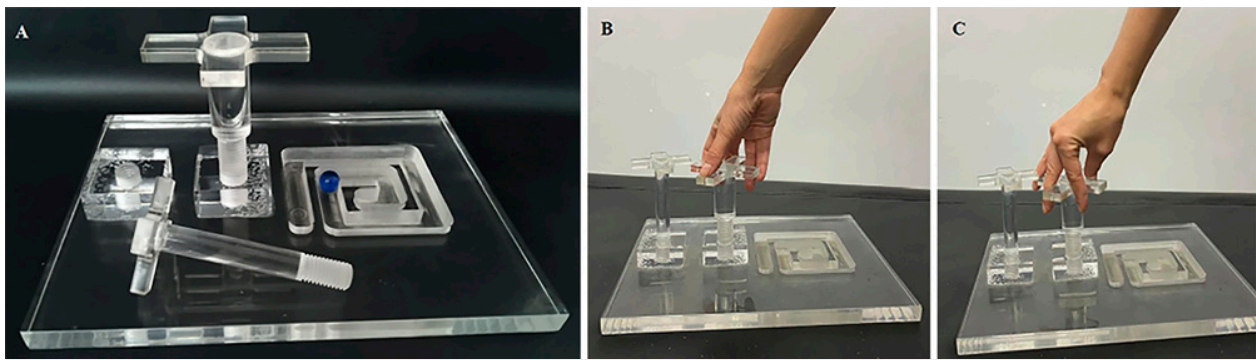

FIG. 2. A: Ad hoc MTD. MTD was manufactured with acrylic to be compatible with fMRI scans. B and C: Upper limb motor function. MTD was planned to perform a continuum medial rotation-cubital deviation movement of the upper limb that combines the qualitative monitoring of distal and proximal muscles in a short period and allows early detection of subtle motion compromise.

and subcortical fibers of the pyramidal tract was performed with conventional flexion-extension tasks.

Resection concludes if the qualitative functional feedback indicates a substantial or an additional decrease in motion during the performance of motor tasks, detailed in MTD description that is corroborated by motor-evoked responses at regular intervals (1 to 3 minutes) or immediately after the detection of an early subtle motion compromise. A reduction of $50 \%$ in the amplitude of MEP regarding a basal recording previous to FCD resection is the optimal goal to conclude the surgery to avoid a permanent deficit. This qualitative feedback cannot be considered as brain mapping because basic principles of brain mapping combine two essential processes that are indispensable and mutually inclusive: function stimulation and effect monitorization. Both stimulation and monitorization only taken as a unit can be considered together as brain mapping.

In epilepsy surgery, mapping can be performed in an intraoperative or extraoperative scenario simultaneously with recording of epileptogenic abnormalities, either through subdural-electrocorticographic or stereo-electroencephalographic recordings. ${ }^{13,17,29,30}$ The main advantage of extraoperative mapping is an increased time available for stimulation. However, this must be balanced against increased risks of infection, bleeding, and higher costs.

In contrast, intraoperative mapping is dynamic, and function can be explored in real time. ${ }^{10,13,31}$ Furthermore, an awake craniotomy provides a unique opportunity to perform a comprehensive functional mapping of the various modalities that integrate a specific function to be preserved. ${ }^{32}$ The patient is both reactive and proactive during the mapping procedures and these are reasons to select this approach when there is no contraindication. In addition, resection of surgical boundaries can be extended in an awake patient even if they are extremely close to eloquent zones with the quantitative and qualitative functional feedback alternative suggested.

This report represents one of the few cases with pure motor strip FCD resection. ${ }^{14,15,23}$ In scenarios similar to this case, we consider that this variation can be useful to improve seizure control and the quality of life of these patients by extending the resection of a more extensive epileptogenic zone minimizing functional damage. Nevertheless, results cannot be generalized from individualized cases and more extensive series are required that represents a limitation of our report. In previous reports, early surgery of motor strip FCDs is recommended to avoid cognitive and social impairment secondary to refractory epilepsy. $7,15,20$

\section{References}

1. Raabe A, Beck J, Schucht P, Seidel K. Continuous dynamic mapping of the corticospinal tract during surgery of motor eloquent brain tumors: evaluation of a new method. J Neurosurg. 2014;120(5): 1015-1024.

2. Szelényi A, Bello L, Duffau H, et al. Intraoperative electrical stimulation in awake craniotomy: methodological aspects of current practice. Neurosurg Focus. 2010;28(2):E7.

3. Landazuri P, Eccher M. Simultaneous direct cortical motor evoked potential monitoring and subcortical mapping for motor pathway preservation during brain tumor surgery: is it useful? J Clin Neurophysiol. 2013;30(6):623-625.

4. Shiban E, Krieg SM, Haller B, et al. Intraoperative subcortical motor evoked potential stimulation: how close is the corticospinal tract? J Neurosurg. 2015;123(3):711-720.

5. Seidel K, Beck J, Stieglitz L, Schucht $P$, Raabe A. The warning-sign hierarchy between quantitative subcortical motor mapping and continuous motor evoked potential monitoring during resection of supratentorial brain tumors. J Neurosurg. 2013;118(2):287-296.

6. Rowland NC, Englot DJ, Cage TA, Sughrue ME, Barbaro NM, Chang EF. A meta-analysis of predictors of seizure freedom in the surgical management of focal cortical dysplasia. J Neurosurg. 2012;116(5):1035-1041.

7. Boex C, Haemmerli J, Momjian S, Schaller K. Prognostic values of motor evoked potentials in insular, precental, or postcentral resections. J Clin Neurophysiol. 2016;33(1):51-59.

8. Jain $P$, Ochi A, Mclnnis $C$, et al. Surgical outcomes in children with bottom-of-sulcus dysplasia and drug-resistant epilepsy: a retrospective cohort study. J Neurosurg Pediatr. 2021;28(3):1-11.

9. Tringali G, Bono B, Dones I, et al. Multimodal approach for radical excision of focal cortical dysplasia by combining advanced magnetic resonance imaging data to intraoperative ultrasound, electrocorticography, and cortical stimulation: a preliminary experience. World Neurosurg. 2018;113:e738-e746.

10. Magill ST, Han SJ, Li J, Berger MS. Resection of primary motor cortex tumors: feasibility and surgical outcomes. J Neurosurg. 2018;129(4):961-972.

11. Southwell DG, Birk HS, Han SJ, Li J, Sall JW, Berger MS. Resection of gliomas deemed inoperable by neurosurgeons based on preoperative imaging studies. J Neurosurg. 2018;129(3):567-575.

12. Rossi M, Sciortino T, Conti Nibali M, et al. Clinical pearls and methods for intraoperative motor mapping. Neurosurgery. 2021; 88(3):457-467. 
13. Rossi M, Conti Nibali M, Viganò L, et al. Resection of tumors within the primary motor cortex using high-frequency stimulation: oncological and functional efficiency of this versatile approach based on clinical conditions. J Neurosurg. Published online August 9, 2019. doi: 10.3171/2019.5.JNS19453.

14. Gopinath S, Roy AG, Vinayan KP, et al. Seizure outcome following primary motor cortex-sparing resective surgery for perirolandic focal cortical dysplasia. Int J Surg. 2016;36(Pt B):466-476.

15. Wang $\mathrm{S}$, Zhang $\mathrm{H}$, Liu $\mathrm{C}$, et al. Surgical treatment of children with drug-resistant epilepsy involving the Rolandic area. Epileptic Disord. 2021;23(2):376-384.

16. McNeal DW, Darling WG, Ge J, et al. Selective long-term reorganization of the corticospinal projection from the supplementary motor cortex following recovery from lateral motor cortex injury. J Comp Neurol. 2010;518(5):586-621.

17. Ostergard TA, Miller JP. Surgery for epilepsy in the primary motor cortex: a critical review. Epilepsy Behav. 2019;91:13-19.

18. Choi SA, Kim SY, Kim H, et al. Surgical outcome and predictive factors of epilepsy surgery in pediatric isolated focal cortical dysplasia. Epilepsy Res. 2018;139:54-59.

19. Crino PB. Focal cortical dysplasia. Semin Neurol. 2015;35(3): 201-208.

20. Sacino MF, Ho CY, Whitehead MT, et al. Repeat surgery for focal cortical dysplasias in children: indications and outcomes. J Neurosurg Pediatr. 2017;19(2):174-181.

21. Zhao B, Zhang C, Wang X, et al. Sulcus-centered resection for focal cortical dysplasia type II: surgical techniques and outcomes. J Neurosurg. 2020;135(1):1-7.

22. Minkin K, Gabrovski K, Karazapryanov P, et al. Awake epilepsy surgery in patients with focal cortical dysplasia. World Neurosurg. 2021;151:e257-e264.

23. Farhat S, Darwish H, Nasreddine W, Salame J, Beydoun A. A surgical case of complete resection of the focal cortical and subcortical dysplasia in the motor cortex. World Neurosurg. 2019;132: 93-98.

24. Ying Z, Wang I, Blümcke I, et al. A comprehensive clinico-pathological and genetic evaluation of bottom-of-sulcus focal cortical dysplasia in patients with difficult-to-localize focal epilepsy. Epileptic Disord. 2019;21(1):65-77.

25. Duffau $\mathrm{H}$. Mapping the connectome in awake surgery for gliomas: an update. J Neurosurg Sci. 2017;61(6):612-630.

26. Byrne RW. Functional Mapping of the Cerebral Cortex: Safe Surgery in Eloquent Brain. 1st ed. Springer; 2016.
27. Saito T, Tamura M, Chernov MF, Ikuta S, Muragaki Y, Maruyama T. Neurophysiological monitoring and awake craniotomy for resection of intracranial gliomas. Prog Neurol Surg. 2018;30:117-158.

28. Hervey-Jumper SL, Berger MS. Maximizing safe resection of low- and high-grade glioma. J Neurooncol. 2016;130(2):269-282.

29. Lehtinen H, Mäkelä JP, Mäkelä T, et al. Language mapping with navigated transcranial magnetic stimulation in pediatric and adult patients undergoing epilepsy surgery: comparison with extraoperative direct cortical stimulation. Epilepsia Open. 2018;3(2):224-235.

30. Young JJ, Coulehan K, Fields MC, et al. Language mapping using electrocorticography versus stereoelectroencephalography: a case series. Epilepsy Behav. 2018;84:148-151.

31. Rossi M, Sani S, Nibali MC, Fornia L, Bello L, Byrne RW. Mapping in low-grade glioma surgery: low- and high-frequency stimulation. Neurosurg Clin N Am. 2019;30(1):55-63.

32. Duffau H. Brain Mapping: From Neural Basis of Cognition to Surgical Applications. Vol. 1. Springer; 2011.

\section{Disclosures}

The authors report no conflict of interest concerning the materials or methods used in this study or the findings specified in this paper.

\section{Author Contributions}

Conception and design: Sandoval-Bonilla, Palmini, Pérez-Reyes, SánchezDueñas. Acquisition of data: Sandoval-Bonilla, Rodríguez-Hernández, Sánchez-Dueñas. Analysis and interpretation of data: Sandoval-Bonilla, Paglioli, De la Cerda-Vargas, Chavez-Herrera, Castro-Prado, PerezCardenas, Sánchez-Dueñas, Lagunes-Padilla. Drafting the article: Sandoval-Bonilla, Palmini, De la Cerda-Vargas, Rodríguez-Hernández, Chavez-Herrera, Pérez-Reyes, Castro-Prado, Perez-Cardenas, LagunesPadilla. Critically revising the article: Sandoval-Bonilla, Palmini, Paglioli, De la Cerda-Vargas, Rodríguez-Hernández, Chavez-Herrera, Pérez-Reyes, Castro-Prado, Perez-Cardenas, Lagunes-Padilla. Reviewed submitted version of manuscript: Sandoval-Bonilla, Paglioli, De la Cerda-Vargas, Chavez-Herrera, Castro-Prado, Perez-Cardenas, Sánchez-Dueñas, Lagunes-Padilla. Approved the final version of the manuscript on behalf of all authors: Sandoval-Bonilla. Administrative/technical/material support: Sandoval-Bonilla, Monroy-Sosa, Rodríguez-Hernández, Chavez-Herrera, Pérez-Reyes. Study supervision: Sandoval-Bonilla, Castro-Prado.

\section{Correspondence}

Bayron A. Sandoval-Bonilla: CMN Hospital de Especialidades, Mexico City, Mexico. bayronsandoval@gmail.com. 